\title{
L'artiste : habitant, résident, touriste. Réflexions à propos de Francis Alÿs
}

The artist: inhabitant, resident, tourist. Reflections on Francis Alÿs

\section{Pascale Riou}

\section{QpenEdition}

\section{Journals}

\section{Édition électronique}

URL : http://journals.openedition.org/tourisme/2073

DOI : 10.4000/tourisme.2073

ISSN : 2492-7503

Éditeur

Éditions touristiques européennes

\section{Référence électronique}

Pascale Riou, «L'artiste : habitant, résident, touriste. Réflexions à propos de Francis Alÿs », Mondes du Tourisme [En ligne], 15 | 2019, mis en ligne le 01 juin 2019, consulté le 30 octobre 2019. URL : http:// journals.openedition.org/tourisme/2073; DOI : 10.4000/tourisme.2073

Ce document a été généré automatiquement le 30 octobre 2019

\section{cc)}

Mondes du tourisme est mis à disposition selon les termes de la licence Creative Commons Attribution - Pas d'Utilisation Commerciale - Pas de Modification 4.0 International. 


\title{
L'artiste : habitant, résident, touriste. Réflexions à propos de Francis Alÿs
}

The artist: inhabitant, resident, tourist. Reflections on Francis Alÿs

\author{
Pascale Riou
}

1 Habiter est une notion fondamentale pour l'étude de l'activité artistique et de l'art, entendu dans son étymologie - ars, artis : manière d'être, manière d'agir. Dans son sens premier, " "Habiter" (wohnen) signifie "être-présent-au-monde-et-à-autrui" » (Paquot et al., 2007, p. 13) ${ }^{1}$. L'action d'habiter comporte ainsi une dimension existentielle. Mais habiter recouvre également un ensemble d'interrelations avec le monde et avec autrui ; avec différents espaces, du Monde au Moi en passant par exemple par l'espace de travail, l'espace domestique, l'espace d'exposition, etc. Ces interactions hommeenvironnement ont également des temporalités, distinctes ou poreuses, rigides ou mouvantes. Le temps est en effet aussi primordial que l'espace pour la problématique de l'habiter envisagée dans le champ artistique. Il permet ainsi d'observer des pratiques, de prendre en compte les rythmes et les déplacements, d'interroger l'œuvre dans ses processus.

2 Selon le canon actuel de la présentation des artistes, Francis Alÿs, «né en 1959 à Anvers, vit et travaille à Mexico $»^{2}$. Mais si l'artiste belge est effectivement installé à Mexico depuis 1986, son activité nous semble emblématique de ce que signifie habiter le monde, et plus précisément de l'habiter en touriste. Artiste de l'action furtive, inutile, poétique et politique - donnée à voir par la trace, souvent photographique ou vidéo -, sa manière d'être artiste et d'agir en tant que tel en fait un habitant singulier, à la fois résident et touriste. Son positionnement artistique et les œuvres qu'il crée et déploie dans et entre différents lieux du globe nous permettent d'interroger les notions d'«habiter» et de «touristique» dans le champ de la création artistique contemporaine. 


\section{Francis Alÿs, habitant, résident, touriste : trois exemples d'œuvres}

3 Francis Alÿs réside à Mexico et, pendant longtemps, s'est considéré comme touriste, dans sa ville de résidence comme dans les autres lieux qu'il a été amené à habiter. Nous reprenons ici la définition et la distinction entre habiter et résider synthétisées par Mathis Stock :

Dans les études contemporaines de géographie urbaine ou de sociologie urbaine, habiter signifie "occuper un logement" ou "résider" (cf. Lelièvre \& LévyVroeland, 1992). Par conséquent, les « habitants » sont ceux qui résident dans un lieu donné. [or,] les êtres humains n'habitent pas seulement un lieu de domicile, ou plus précisément : n'habitent pas seulement lorsqu'ils résident... En effet, c'est par la pratique des lieux que l'individu les habite » (2004).

4 Et cette pratique peut être multiple, notamment touristique. Le touriste étant l'individu qui « associe un déplacement à une pratique de re-création » (Darbellay et Stock, 2012).

5 Trois œuvres sont emblématiques de cette pratique de l'espace déployée par Francis Alÿs, mêlant éthique et politique, questionnant les notions d'habiter et de résider et faisant intimement dialoguer artistique et touristique : Turista (1995), The Loop (1997) et Looking Up (2001).

Turista, 1995, Mexico, action documentée (fig. 1) 
Fig. 1

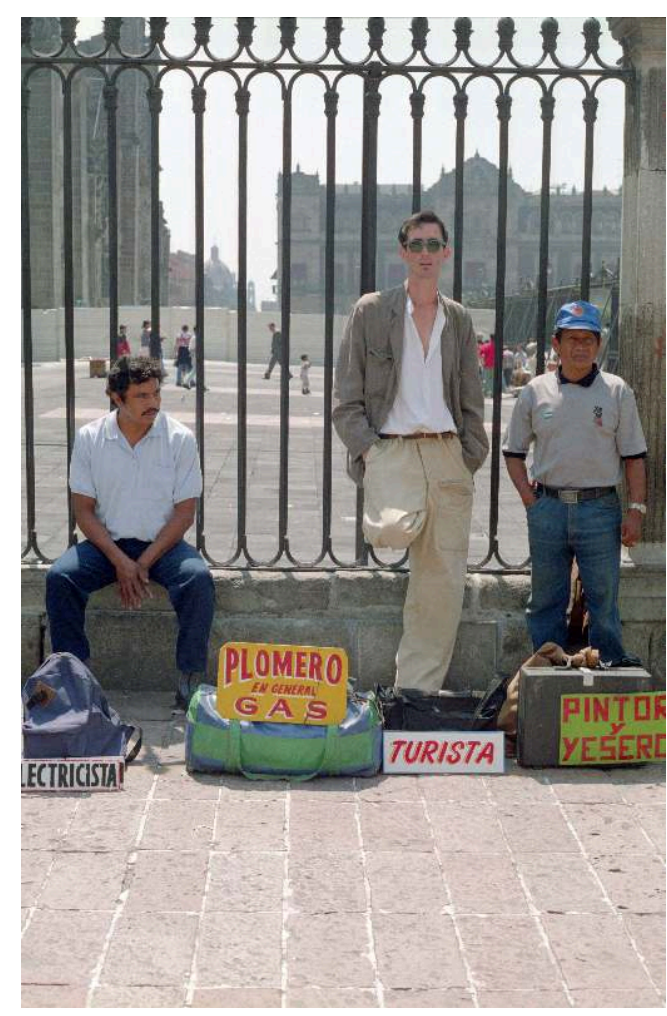

Francis Alÿs

Turista

Mexico City, 1994

Photographic documentation of an action

Courtesy David Zwirner, New York/London

Pour cette action, Francis Alÿs se rend sur la place de la Constitution, place centrale de Mexico, très fréquentée, autant par les touristes que par les habitants. C'est ainsi le lieu où les travailleurs de diverses professions ont coutume de s'installer les uns à côté des autres, proposant leurs savoir-faire et services à qui veut les employer. Alignés le long d'une grille, chacun a une pancarte qui présente ses compétences ou son métier: « électricien ", " plombier », " peintre-plâtrier », etc. L'artiste prend place dans la file des demandeurs d'emploi et arbore quant à lui une pancarte sur laquelle est inscrit «touriste». L'action est archivée par une photographie couleur montrant l'artiste entre deux hommes, nonchalamment adossé aux barreaux en fer forgé, les mains dans les poches, des lunettes de soleil sur le nez. Les travailleurs se postent sur l'extérieur de la place afin de rester proches de la route et visibles des passants, piétons comme motorisés. Le touriste Francis Alÿs se positionne quant à lui selon un angle de vue oblique par rapport à ceux les plus souvent choisis par les touristes, mettant notamment la cathédrale métropolitaine de Mexico à l'honneur. La prise de vue de l'action reflète les manières de faire privilégiées de cet artiste, l'obliquité et la furtivité.

7 L'artiste incarne ainsi un touriste lambda et propose aux passants intéressés de l'embaucher en tant que tel. Il fait du tourisme un métier. "Touriste » le définit alors socialement et l'individualise par rapport aux demandeurs d'emploi qui l'entourent. Turista fonctionne comme une déclaration de principe : c'est en touriste que l'artiste se présente et se représente, aux hommes qui l'entourent comme à ceux qui verront de cette action la trace photographique. C'est en touriste qu'il œuvre, qu'il prend part au 
monde et l'habite. Cette œuvre, comme d'autres qui suivront, témoigne donc de son engagement à faire du déplacement " une raison d'être, un acte social et politique à part entière, un travail. » (Davila, 2002, p. 18).

\section{The Loop, 1997, action documentée (fig. 2)}

Fig. 2

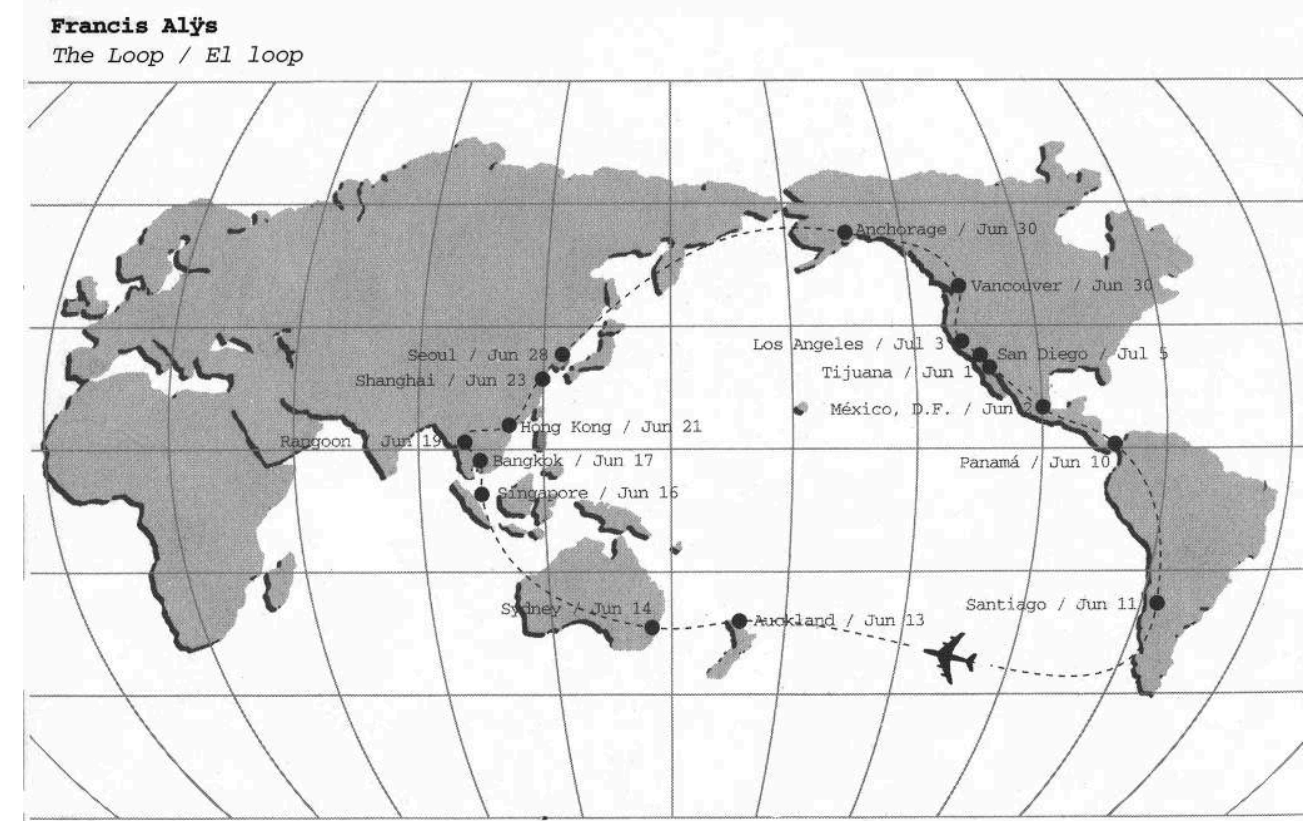

\section{iNSITE97}

Francis Alÿs

Tijuana-San Diego, 1997

Ephemera of an action

Courtesy David Zwirner, New York/London

Deux ans plus tard, à l'occasion de l'exposition In Site 97, Francis Alÿs passe un contrat de «touriste professionnel » avec les organisateurs, les villes de Tijuana (Mexique) et San Diego (États-Unis), à une trentaine de kilomètres l'une de l'autre. Il réalise ensuite The Loop en allant de Tijuana à San Diego en une boucle de trente-cinq jours autour du Pacifique, par avion. Son objectif est de relier les deux villes sans traverser la frontière qui les sépare. L'action est finalement visible dans l'exposition par l'édition de cartes postales à disposition du public; format léger s'il en est, également amené à voyager, qui symbolise l'échange autant que les vacances. Cette action va à l'encontre de la rationalité, puisque l'artiste n'emprunte pas le chemin le plus court du point de départ au point d'arrivée, mais le " meilleur », le plus adéquat pour lui et le message qu'il veut véhiculer. Ainsi, en ne traversant pas la frontière mais en la contournant, il la met en évidence.

Plus largement, le statut de nomade est, pour Francis Alÿs, un statut officiel ${ }^{3}$. Un statut efficient car le nomadisme est envisagé comme «l'état même de la situation artistique " (Davila, 2002, p. 19). Il est artiste ici et ailleurs, et tout le temps, en recherche perpétuelle. Il s'agit alors de "construire un processus à partir d'un mouvement, d'inventer un agencement qui sera avant tout un trajet, d'inventer un 
agencement comme trajet et ainsi de donner forme au mouvement» (Davila, 2002, p.35). L'artiste lie ainsi, par sa pratique des lieux et de l'espace, le poétique au politique. Avec The Loop, il ne propose pas seulement un grand tour en touriste, mais aussi un détour et un détournement critique de la situation socio-politique entre le Mexique et les États-Unis - la pertinence de cette œuvre est toujours valable près de vingt ans plus tard.

\section{Looking Up, 2001, vidéo, 3 min $33^{4}$. (fig. 3)}

Fig. 3

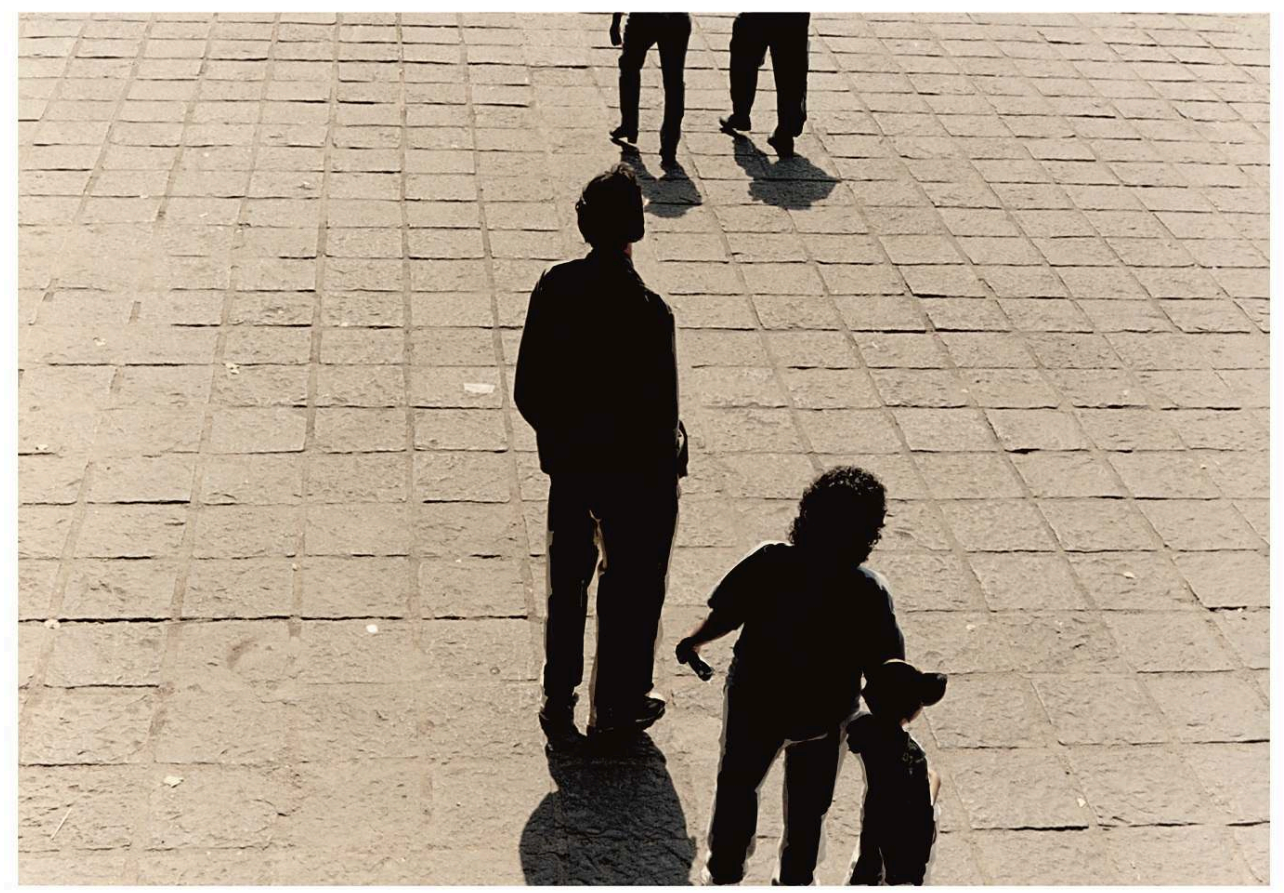

Francis Alÿs

Looking Up

Mexico City, 2001

In collaboration with Rafael Ortega

Video (4 minutes) and photographic documentation of an action

Courtesy David Zwirner, New York/London

La figure du touriste comme promeneur qui déambule, qui prend le temps de vaquer sans occupation prédéfinie et d'observer son environnement se retrouve particulièrement dans cette vidéo. Le lieu de l'action est à nouveau une place publique de Mexico, celle de Santo Domingo, filmée en plan fixe et en plongée sur ses pavés. Deux passants traversent le champ, puis Francis Alÿs apparait. Il marche et s'arrête au centre du plan, n'esquisse pas de mouvement particulier, ne fait apparemment rien si ce n'est regarder attentivement quelque chose au loin, hors champ. Rapidement, des passants le remarquent et s'arrêtent aussi, cherchant à voir ce que regarde l'artiste. Ces gens, qui semblent suivre une trajectoire décidée, font alors une pause dans leur marche utile et peut-être quotidienne, trajet entre lieu de travail et domicile par exemple. Après quelques dizaines de secondes, l'artiste part, comme il est arrivé, 
laissant les badauds qui s'étaient regroupés autour de lui reprendre leur marche et leurs activités.

L'artiste effectue un geste simple, discret, et cherche avec humour la participation des passants curieux. Pendant un court moment, chacun a oublié son objectif. Ici, la figure du touriste oisif propose une expérience à la fois personnelle et commune. Par des gestes banals, l'artiste met en œuvre des réflexions sur le quotidien, le rythme de vie et de travail, l'individu et le groupe. Une telle pratique nous semble tendre vers une intention, commune à d'autres artistes : «trouver quelque chose à faire pour bâtir jour après jour [...] ce que l'on pourra appeler une œuvre [et] faire de ce temps passé un

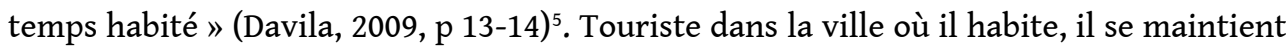
pourtant hors des habitudes, de la routine et reste disponible aux détails et surprises, quitte à les inventer : «Marcher est pour Alÿs l'occasion d'expérimenter le réel, jusqu'à la fiction» (Dressen, 2003, p. 145). Plus largement, la mobilité est pour l'artiste une manière de créer.

À travers ces trois exemples, le cas de Francis Alÿs nous amène à nous demander comment l'artiste peut habiter en touriste et comment il peut interroger le concept particulier de résidence artistique.

\section{L'artiste habite en touriste}

13 La racine du mot tourisme est tour: le touriste se déplace suivant un mouvement presque circulaire, pour revenir à son point de départ ${ }^{6}$. On peut également appréhender le tourisme par la dimension de vacances, de loisir au singulier et de loisirs au pluriel, d'un hors travail quantitativement de plus en plus important, qui appelle à l'appropriation de ce «temps à soi » (Viard, 2006, 2015), voire d'un hors quotidien ou plutôt d'un autre quotidien, fait à la fois d'ordinaire et d'extraordinaire (Vacher et Vye, 2012). Or, être ouvert, se déplacer - physiquement comme intellectuellement-, s'adapter sont des manières d'être et de faire inhérentes à l'activité touristique qui caractérisent également l'activité artistique. Chez Francis Alÿs, trois facettes de l'artiste habitant en touriste semblent émerger et croiser ces notions de mobilité, de dépaysement et de loisir.

D'une part, l'artiste apparaît comme un touriste-marcheur. En effet, l'atelier principal de Francis Alÿs ne nous semble pas être circonscrit entre quatre murs, mais s'étendre à la ville de Mexico toute entière. Dans une présentation de l'artiste, Anne Dressen relate :

Alÿs aime à dire qu'il est entré dans l'art en découvrant Mexico, paradigme monstrueux de l'urbanité et de ses flux. Il quitta alors l'architecture pour mieux se plonger dans la ville. Depuis, les grandes mégalopoles du monde sont devenues le terrain privilégié de ses « Paseos » (marches). [...] les performances de Francis Alÿs - toutes fondées sur le déplacement - sont ancrées dans la ville envisagée comme un laboratoire » (2003, p. 145).

Certes l'artiste a un studio, proche du centre historique de Mexico, mais il fait de son environnement immédiat son atelier, d'autant que son activité l'a amené à transformer son studio en un bureau et un lieu de stockage ${ }^{7}$. Par le déplacement physique hors les murs, l'artiste élargit le lieu de son activité artistique et se fabrique les conditions propices à cette pratique. Francis Alÿs revendique par ailleurs son statut de passant, 
dont la quête constante est de toujours pouvoir se situer dans un environnement mobile et changeant ${ }^{8}$.

$\mathrm{Au}$ sein de la capitale mexicaine, et ailleurs au gré de ses projets, la marche, en tant que déplacement, déambulation et flânerie, fait partie de la pratique touristico-artistique de Francis Alÿs9. En cela, il participe à une histoire de l'art contemporain dans laquelle la notion d'atelier est redéfinie (Davidts et Paice, 2009). Jean-Pierre Criqui note, pour sa part, qu'à partir de la fin des années 1960,

Ce qui commence alors à se défaire, c'est la notion de l'atelier entendu comme lieu central de production à partir duquel l'art est acheminé vers d'autres centres - les galeries, les musées et les biens nommés « centres d'art »... (2002, p. 184)

De Richard Long et les artistes du Land Art à Mathias Poisson et ses "graphies du déplacement ", en passant par Gabriel Orozco, flâneur refusant le principe d'atelier fixe, ou encore Céline Ahond et ses visites performées, nombreux sont les artistes qui se sont emparés de cette pratique de la marche hors des lieux traditionnels de production et de monstration de l'art.

Par une activité autrement localisée, une manière de faire sans centralité - ou du moins avec plusieurs centres selon les circonstances de la pratique -, l'artiste touriste se rend disponible à l'occasion et concourt à déplacer le postulat de l'habiter de son sens premier d'« être dans l'espace " vers la problématique de la pratique, c'est-à-dire « faire avec de l'espace » (Stock, 2004). Nous pouvons nous référer ici à une autre vidéo de Francis Alÿs, caractéristique de ce positionnement: Sometimes Making Something Leads to Nothing (Mexico, 1997, 9 min 54), dans laquelle on suit l'artiste à travers les rues de Mexico, poussant un bloc de glace sur le sol jusqu'à ce que celui-ci ait entièrement fondu ${ }^{10}$. Le fondement de la pratique de cet artiste est bien d'œuvrer avec de l'espace espace entendu comme lieu pratiqué, soumis à la temporalité (Certeau, 1990). Il déclare par ailleurs dans une note personnelle : "As long as I'm walking, I'm not thinking " [Tant que je marche, je ne pense pas] (Medina, 2014, n.p.) et, dans un entretien, ce qui semble paradoxal : « The initial concept for a project often emerges during a walk » [Le concept initial d'un projet émerge souvent pendant une marche] (Lütgens, 2004, p. 41). Ce qui pourrait à première vue être une contradiction s'avère être le nœud de la pratique de Francis Alÿs : ce n'est pas d'une pensée, d'une volonté ou d'une prévision, mais du corps et de cette observation participante située que naît l'intentionnalité de l'artiste et son œuvre ${ }^{11}$. L'espace de la pratique de l'artiste, son terrain, est alors à envisager comme "espace vu du corps» (Volvey, 2000), ou plutôt comme lieu d'interaction corps-espace ${ }^{12}$.

D'autre part, l'artiste en touriste agit comme un révélateur. Les actions de Francis Alÿs nous semblent pointer, mettre à jour des situations, géographiques, sociales ou politiques. Certes, les performances en elles-mêmes sont furtives, non démonstratives, mais les traces documentaires - par exemple, la photographie, comme pour Turista, ou l'édition, dans le cas de The Loop - en permettent l'archive, l'exposition et surtout l'existence: «in his work, the trace is the practice» [dans cet œuvre, la trace est la pratique] rappelle Annelie Lütgens (2004, p. 53). Peintures, dessins, photographies ou objets trouvés sont produits et montrés, ainsi que des vidéos, forme privilégiée dans la pratique de l'artiste. Cette forme permet notamment de rendre compte de l'action dans son processus et sa durée, même si la grande majorité est constituée d'une succession de plans, donc d'ellipses. Une diffusion large en est ensuite proposée via Internet ${ }^{13}$. 
20 The Green Line (Jérusalem, 2004, 17 min 41) est emblématique de l'engagement de l'artiste-touriste, marcheur et révélateur ${ }^{14}$. Introduite par la mention, en anglais, «Parfois faire quelque chose de poétique peut devenir politique et parfois faire quelque chose de politique peut devenir poétique ", la vidéo rend compte d'une performance réalisée en juin 2004 à Jérusalem. L'artiste marche sur 24 kilomètres le long de la ligne verte délimitant l'État d'Israël selon le tracé de l'Organisation des Nations unies (ONU) de 1948. Durant sa marche, l'artiste tient dans sa main un pot de peinture verte percé d'un trou, laissant couler son contenu au sol et traçant dans l'espace le trait de crayon jadis apposé sur une carte. La réflexion est, là encore, effectivement politique et poétique, questionnant d'une manière symbolique autant que dérisoire le phénomène frontalier au cœur d'un des territoires où il est le plus problématique. L'engagement de l'artiste ne relève pas de la démonstration, ni du militantisme, et c'est ce qui nous semble être sa force. Parce que l'artiste est en perpétuel déplacement, il est perpétuellement déplacé, attentif au monde qu'il habite et dont il rend compte.

Enfin, un troisième terme peut qualifier et préciser la figure du touriste incarnée par Francis Alÿs : joueur. Art et jeu sont souvent des corollaires, relatifs parfois au temps libre, souvent au plaisir. Cela revient à une acception péjorative du mot touriste, qui se rapproche de l'acception péjorative du mot amateur, celui qui fait mal, sans sérieux : "Amateur, personne qui s'intéresse aux choses avec curiosité mais d'une manière superficielle »; «En touriste. En amateur, en dilettante ${ }^{15}$. Or, certains artistes interrogent les notions de professionnalisme et d'amateurisme afin de mieux gommer des catégories trop strictes pour prendre en compte les spécificités de l'activité artistique (Freidson, 1986; Nicolas-Le Strat, 2000). En se présentant comme touriste professionnel, Francis Alÿs joue avec les définitions, les valeurs, les représentations sociales et culturelles de l'artiste comme du touriste. Pour Michel Guérin, la situation sociale marginale de l'artiste remonte à l'Antiquité : «Platon ne voit, dans la Cité juste, aucune possibilité qu'une place honorable soit consentie à ce que nous identifions comme activité artistique » (2007, p. 45). Ce qui condamne l'artiste, c'est qu'il n'occupe pas de place précise, de fonction assignée; il ne peut alors "que jeter le trouble » (Guérin, 2007, p. 45). Le touriste est, lui aussi, celui qui parfois n'a pas sa place, n'est pas «chez lui ». Francis Alÿs, revenant sur Turista, déclare avoir réalisé cette œuvre à un moment où il souhaitait être honnête avec sa situation d'étranger - habitant mais déplacé (Diserens, 2004, p. 115) ${ }^{16}$. En jouant avec les cadres et les contextes et en faisant jouer les cadres et les contextes, l'artiste tente d'inventer ses propres règles. Il n'est pas étonnant alors qu'une série de vidéos, en cours depuis 1999, s'attache aux jeux d'enfants. Francis Alÿs, accompagné parfois de collaborateurs, rend compte de divers jeux universels : faire des ricochets (Tanger, 2007), sauter à l'élastique (Paris, 2008), faire rouler un pneu avec un bâton (Bamiyan, 2010), jouer à " pierre, feuille, ciseaux » (Mexico, 2013) ${ }^{17}$. Le cadrage de l'image est le plus souvent concentré sur l'action et les gestes, le caméraman, proche des joueurs, voire parmi eux. Le regardeur, comme l'artiste, observe l'action de près, suit les gestes et mouvements des joueurs, les accompagne.

22 L'œuvre de Francis Alÿs est ainsi empreinte d'une "poétique de l'habiter » (Ingold, 2012, p.19). De l'habiter en touriste découlent une attention et des gestes qui constituent un espace-temps de la pratique singulier. Cela nous amène à interroger une notion qui n'est pas abordée explicitement par l'artiste mais qui nous paraît néanmoins 
fondamentale dans son œuvre, en faisant dialoguer la notion d'habiter en touriste avec celle de résider en artiste.

\section{L'artiste résident : touriste et habitant ?}

Rappelons tout d'abord qu'une distinction est établie par Georges-Hubert de Radkowski entre les notions de résidence et d'habitat. Cette distinction tient notamment au fait que la résidence est un espace social, et non un espace physique, comme l'est l'habitat ${ }^{18}$ - ce qui n'empêche pas de l'habiter: "C'est parce qu'elle est le lieu où l'homme est présent pour les autres que la résidence peut devenir sienne (son chez soi)» (Radkowski, 2002, p.46). La résidence, dans le champ artistique, répond à cette caractéristique : un espace-temps socialement défini, hors habitat et hors habitudes, que l'artiste habite temporairement. Les résidences artistiques peuvent prendre plusieurs formes, souvent celles d'ateliers provisoires, d'espaces de vie et de travail ponctuels, parfois attribués sur appel à projets ou assortis d'une obligation de production, d'exposition voire de médiation. La résidence déplace momentanément l'artiste et sa pratique et peut être une occasion d'émergence de formes, d'expérimentations, de rencontres. En faisant de Mexico son atelier, son laboratoire, Francis Alÿs nous semble faire de sa ville de résidence sa résidence artistique permanente. "Pour Alÿs, les lieux qu'il fréquente et où il a vécu ont vocation à déconcerter" (Monsiváis, 2005, p. 17). Autrement dit, les lieux en tant que tels le déplacent et déplacent sa pratique. L'écrivain Carlos Monsiváis revient sur le lien de l'artiste à sa ville de résidence en ces termes : «Dès son arrivée à Mexico, Francis Alÿs s'est intéressé au Centre historique ", notamment à son " potentiel esthétique » et au «devenir du Centre»(2005, p. 16-17). Ce centre est l'«espace de prédilection» de Francis Alÿs, en tant qu' « état des choses » (Monsiváis, 2005, p. 55) ; un état des choses en constante évolution, constitué de repères mouvants, avec lesquels faire. Ainsi, la localisation de son studio dans le centre est primordiale. En 2004, l'artiste se fabrique un temps de travail particulier, lié à une exposition au Kunstmuseum Wolfsburg: Walking Distance from the Studio; soit l'exploration volontairement restreinte à un périmètre donné de 10 blocks autour de son studio (Alÿs, 2004); une expérimentation fine d'un environnement immédiat destinée à familiariser l'artiste au quotidien et à ses surprises (van Tuyl, dans Alÿs, 2004). Après quelques années de résidence, d'arpentage et d'observation participante de Mexico, Francis Alÿs touriste-résident-habitant ne semble plus un outsider, sans pour autant être tout à fait un insider ; l'évolution des lieux et sa propre mobilité l'empêchent de s'installer - au sens strict comme au figuré. Il continue d'en faire le tour car, précisément, il ne peut pas en faire le tour.

Par ailleurs, la notion de résidence artistique recoupe celles de réseau et d'échange, ce qui met en perspective la remarque de Georges-Hubert de Radkowski :

... habiter ne désigne plus ici résider [dans l'urbain] mais - virtuellement ou actuellement - communiquer (dont le circuler est l'aspect le mieux connu). Le citadin-résident est un abonné à ces points nodaux de l'espace-réseau ; sa résidence, un branchement sur ce réseau. (2002, p. 146)

L'artiste en résidence réside et communique, prend place et se déplace au sein d'un réseau. Pour Francis Alÿs, ce réseau semble être autant urbain que rural, autant individuel que social, autant artistique qu'humain; se développant au gré des projets, 
marches, collaborations - les cas que nous évoquons n'en étant que quelques exemples parmi les plus pertinents.

Dans ses "Propos sur les voyages », de 1905, l'écrivain allemand Hermann Hesse pose déjà, sans la formuler ainsi, la problématique de l'habiter comme préalable à l'activité artistique :

... vivre quelque chose d'intéressant n'est possible que dans des lieux avec lesquels nous entretenons une relation particulière. (2002, p. 31)

27 Ainsi, la relation particulière se tisse dans un lieu que l'on fréquente, qui nous est familier. Cela sous-entend que le voyage doit être l'exception dans un quotidien sédentaire. À moins que le voyage, ou la promenade, ne puisse se vivre au sein même de son propre écosystème et de son agencement. C'est dans cette seconde conception de l'habiter que parait se placer Francis Alÿs, sa mobilité touristico-artistique l'emmenant en premier lieu à faire œuvre dans et avec l'environnement immédiat qu'il a choisi : le centre historique - et touristique - de Mexico.

La résidence artistique est en effet envisagée par les artistes comme une pratique de l'habiter sans cesse renouvelée; le paradoxe étant qu'habiter signifie être et faire en familier et non en habitué. Car l'habitant peut ne pas prendre conscience de ce que le touriste remarquera - et vice versa. L'artiste se situe alors entre deux, se nourrit des deux états d'esprit, provoque des relations particulières à son environnement tout en restant ouvert, à l'affût, perméable à la découverte et au dépaysement, qui favorisent eux aussi l'activité artistique.

Pour conclure, les actions de Francis Alÿs rendent compte d'une pratique d'un espacetemps, c'est-à-dire d'interactions dans et avec cet espace et ce temps au sein d'un réseau de mobilité et d'échange avec cet environnement et ceux qui le peuplent. Son exemple engage à " prendre au sérieux le déplacement comme un mode d'habiter en soi : le passant est aussi un habitant, mais un habitant qui ne cesse de sortir d'un régime de familiarité aux choses et aux gens » (Fort-Jacques, 2007, p. 253). Francis Alÿs, touriste, résidant où l'emmène son activité, donc habitant.

Plus largement, nous observons, dans les quelques cas exemplaires cités, que les figures de l'habitant, du touriste et du résident s'entremêlent pour fabriquer la figure de l'artiste et ses liens avec son environnement immédiat - qu'on pourrait appeler son écosystème (de oikos, foyer et systêma, ensemble) - et son environnement plus large géographique, social, politique - au travers d'interactions essentielles à son activité. Par sa pratique, Francis Alÿs semble faire la synthèse entre les deux types identifiés par Anne Volvey et Mathis Stock: l'« habitant-concepteur des lieux» qu'est l'artiste et l'« habitant-"expérienceur" des lieux » qu'est le touriste (Stock et Volvey, 2014). C'est en effet par l'empirisme de l'action physique que l'artiste développe sa manière de penser et d'être. Habiter, en artiste-touriste, prend bien alors une dimension esthétique et éthique. Comme le rappelle Henri Maldiney :

31 Ethos en grec ne veut pas dire seulement manière d'être mais séjour. L'art ménage à l'homme un séjour, c'est-à-dire un espace où nous avons lieu, un temps où nous sommes présents - et à partir desquels, effectuant notre présence à tout, nous 
communiquons avec les choses, les êtres et nous-mêmes dans un monde, ce qui s'appelle habiter. (2012, p. 202)

L'artiste touriste-résident-habitant met ainsi en œuvre une écosophie, littéralement une sagesse de l'habiter, telle qu'elle a pu être pensée par Arne Næss ou Félix Guattari (Næss, 2013 (1989) ; Guattari, 1989, 2013). Pour les deux philosophes, il s'agit de développer un système ad hoc, donc personnel, qui permette de lier théorie et pratique, l'« être-avec » et le «faire-avec » l'espace-temps et ce qui le constitue. Dans le cas de Francis Alÿs, cela passe par des gestes spécifiques, souvent discrets - marcher, regarder en l'air, prendre l'avion -, une approche poétique des mécanismes sociaux et politiques - déplacements, loisir, travail, frontières -, un engagement du corps dans l'espace et le temps - par des actions physiques documentées. Cela lui permet une constitution de son être et de sa pratique, dans son propre milieu et par son propre milieu, et, réciproquement, de fabriquer les lieux qu'il habite par sa manière d'être et de faire minimale, discrète mais effective. Une sagesse de l'habiter en touriste.

\section{BIBLIOGRAPHIE}

Francis ALŸs, Walking Distance from the Studio, Kunstmuseum Wolfsburg, 2004.

Jean-Pierre CRIQUI, Un trou dans la vie. Essais sur l'art depuis 1960, éditions Desclée de Brouwer, 2002.

Frédéric DARBELLAY et Mathis STOCK, «Penser le touristique : nouveau paradigme ou interdisciplinarité ? ", EspacesTemps.net, 2012, [https://www.espacestemps.net/articles/penserle-touristique-nouveau-paradigme-ou-interdisciplinarite/].

Michel de CERTEAU, L'invention du quotidien, 1. arts de faire, « folio essais », Gallimard, 1990.

Wouter DAVIDTS et Kim PAICE (dir.), The Fall of the Studio: Artists at Work, coll. « Antennae », Valiz, 2009.

Thierry DAVILA, In extremis. Essais sur l'art et ses déterritorialisations depuis 1960, coll. « Essais », éditions La lettre volée, 2009.

Thierry DAVILA, Marcher, créer. Déplacements, flâneries, dérives dans l'art de la fin du XXe siècle, éditions du Regard, 2002.

Corinne DISERENS, «Conversation », Francis ALŸS, Walking Distance from the Studio, Kunstmuseum Wolfsburg, 2004.

Anne DRESSEN, « Francis Alÿs », dans Laurence BOSSÉ et Hans Ulrich OBRIST (dir.), Déplacements, Musée d'Art Moderne/Onestar press, 2003.

Eliot FREIDSON, «Les professions artistiques comme défi à l'analyse sociologique ", Revue française de sociologie, vol. 27, n 3, 1986 [http://www.persee.fr/doc/

rfsoc_0035-2969_1986_num_27_3_2324].

Félix GUATTARI, Qu'est-ce que l'écosophie ?, éditions Lignes/IMEC, 2013.

Félix GUATTARI, Trois écologies, éditions Galilée, 1989. 
Michel GUÉRIN, L'artiste ou la toute-puissance des idées, coll. « Théorie et pratique des arts », Publications de l'Université de Provence, 2007.

Hermann HESSE, « Propos sur les voyages » (1905), dans L'art de l'oisiveté, Calmann-Lévy, 2002.

Tim INGoLD, Faire. Anthropologie, archéologie, art et architecture, Dehors, 2017.

Tim INGOLD, « Culture, nature et environnement », Tracés, n 22, 2012, [http://traces.revues.org/ 5470].

Olivier LAZZAROTTI, Habiter le monde, coll. « Documentation photographique », La Documentation française, 2014.

Annelie LÜTGENS, « Francis Alÿs and the Art of Walking », dans Francis ALŸs, Walking Distance from the Studio, Kunstmuseum Wolfsburg, 2004.

Henri MALDINEY, « L'esthétique des rythmes » (1967), dans Regard parole espace, éditions Cerf, 2012.

Cuauhtémoc MEDINA, dans Francis Alÿs, Pacing, Ivorypress, 2014.

Carlos MONSIVÁIs, Le centre historique de Mexico, images de Francis detto ALŸs, Turner/Musée des Beaux-Arts de Nantes, 2005.

Arne NAESS, Écologie, communauté et style de vie, Dehors, 2013 (1989).

Pascal NICOLAS-LE STRAT, Mutations des activités artistiques et intellectuelles, coll. « Logiques sociales », L'Harmattan, 2000.

Thierry PAQUOT, Michel LUSSAUlt et Chris younÈs (dir.), Habiter, le propre de l'humain. Villes, territoires et philosophie, coll. « Armillaire », La Découverte, 2007.

Georges-Hubert de RADKOWSKI, Anthropologie de l'habiter, PUF, 2002.

Mathis sтоск, «L’habiter comme pratique des lieux géographiques », EspacesTemps.net, 2004 [https://www.espacestemps.net/articles/habiter-comme-pratique-des-lieux-geographiques/].

Mathis STоск et Anne VOLVEY, « Le touriste et l'artiste, l'impossible rencontre ? », dans Marcel HENRY et Raphaël BRUNNER (dir.), Triennale d'art contemporain Valais 2014 - Reader, Benteli, 2014.

LUC VACHER et Didier VYE, «Penser l'habiter à travers les pratiques des lieux touristiques par les excursionnistes, touristes et résidents ", dans Brigitte FRELAT-KAHN et Olivier LAZZAROTTI (dir.), Habiter. Vers un nouveau concept ?, Armand Colin, coll. « Recherches », 2012.

Jean VIARD, Éloge de la mobilité. Essai sur le capital temps libre et la valeur travail, coll. « Essai », éditions de l'Aube, 2006.

Jean VIARD, Le triomphe d'une utopie. Vacances, loisirs, voyages. La révolution des temps libres, coll. « Monde en cours », éditions de l'Aube, 2015.

Anne volvey, « Entre l'art et la géographie, une question (d')esthétique », Belgeo, n 3, 2014

[http://journals.openedition.org/belgeo/13258 ; DOI : 10.4000/belgeo.13258].

Anne volvey, « L'espace vu du corps », dans Jacques lévy et Michel LuSSAUlt (dir.), Logiques de l'espace, esprit des lieux. Géographies à Cerisy, Belin, 2000.

\section{NOTES}

1. L'expression est due à Martin Heidegger. 
2. Cf. http://16fdn9ufhox41fwkc1azw8b1bio.wpengine.netdna-cdn.com/wp-content/uploads/ 2012/05/Francis-Aly\%CC\%88s-CV-2016-3.pdf (consulté le 9 janvier 2017).

3. Nomade étant compris comme mobile et la mobilité touristique comme une manière d'être nomade parmi d'autres. Cf. Rémy KNAFOU (dir.), La planète nomade. Les mobilités géographiques d'aujourd'hui, Belin, 1998: la mobilité n'est plus l'exception à des styles de vie fondés sur l'immobilité mais au contraire des pratiques courantes voire quotidiennes ; Olivier LAzZAROTTI, Habiter le monde, La Documentation française, coll. « Documentation photographique », 2014, p. 4.

4. https://vimeo.com/130939419 (consulté le 9 janvier 2017).

5. L'historien de l'art écrit ces mots à propos de la pratique du peintre On Kawara (1933-2014), une activité quotidienne où le temps est à la fois un sujet, un outil et une matière. Nous les reprenons ici car Francis Alÿs paraît également habiter l'espace comme le temps et bâtir son œuvre par cet habiter.

6. Cf. la définition du Centre national de ressources textuelles et lexicales (Cnrtl) : http:// www.cnrtl.fr/definition/tour (consulté le 9 janvier 2017).

7. Cf. Alÿs, 2004 : « The artist makes his immediate environment his studio » (p. 13) ; « ... I spend a lot of time walking around the city. As my collaboration with craftsmen increases, my studio becomes something between a storage space and an office » (p. 41).

8. « As an artist, my position is akin to that of a passer-by, constantly trying to situate myself in a moving environment " (Lütgens, 2004, p. 41).

9. La marche de Francis Alÿs se distingue en revanche de la dérive situationniste en tant que «technique du passage hâtif à travers des ambiances variées » (Internationale Situationniste, $\mathrm{n}^{\circ} 1$, juin 1958).

10. Cf. http://francisalys.com/sometimes-making-something-leads-to-nothing/ (consulté le 9 janvier 2017).

11. Sur l'observation participante, en tant que "manière de connaitre de l'intérieur ", voir: Ingold, 2017, p. 28.

12. Anne Volvey défend également l'idée du terrain, du géographe comme de l'artiste, en tant que champ (field) et pratique (work) (Volvey, 2014); ce qui correspond bien au rapport entre lieu et activité artistique entretenu par Francis Alÿs, pour lequel l'habiter est bien constitué de spatialité et de relationnalité.

13. Les vidéos de l'artiste sont sur son site web et certaines plateformes vidéos en ligne, disponibles au téléchargement et au partage pour un usage non commercial et hors cadre d'exposition.

14. Cf. http://francisalys.com/the-green-line/ (consulté le 9 janvier 2017).

15. Définition du Cnrtl : http://www.cnrtl.fr/definition/touriste (consulté le 9 janvier 2017).

16. « ... at the time, it was an honest account of my situation, of my status. [...] Now things are different. In spite of my disguise as a foreigner, a stranger, I have been here longer than many of my neighbors, and perhaps I have already become a local character for them... » (Diserens, 2004, p. 115).

17. Cf. http://francisalys.com/category/children-games/ (consulté le 9 janvier 2017).

18. Selon Olivier Lazzarotti, le concept de l'habiter est plus complexe que le verbe habiter, assimilé à «se loger », mais le terme « habitat » peut conserver son sens restrictif de logement (Lazzarotti, 2014). 


\section{RÉSUMÉS}

En 1995, l'artiste belge Francis Alÿs s'exposait sur une place de Mexico avec une pancarte: «Turista ». En 1997, il réalisait The Loop, ralliant Tijuana à San Diego par un tour du Pacifique. En 2001, il s'arrêtait de marcher pour regarder en l'air quelques minutes. Se revendiquant touriste professionnel, il n'a de cesse de questionner le monde à travers l'observation fine de son environnement, dont il rend compte par la métaphore ou la parabole. Figure exemplaire, il nous permet d'interroger l'habiter et, plus précisément, l'habiter en touriste, dans le champ de la création artistique contemporaine. À cette notion s'ajoute celle de résidence, très usitée dans le contexte artistique, qui est ici à questionner. L'artiste touriste-résident-habitant met en œuvre des manières d'être et d'agir autant poétiques que politiques et propose ainsi une certaine sagesse de l'habiter.

In 1995, the Belgian artist Francis Alÿs exhibited himself on a square in Mexico City with a sign: "Turista". In 1997, he did The Loop, rejoining Tijuana to San Diego around the Pacific. In 2001, he stopped walking to look in the air for a few minutes. Claiming himself a professional tourist, he never stops questioning the world through the fine observation of his environment, which he reports through metaphor or parable. As an exemplary figure, he allows us to question the dwelling, and more precisely to dwell as a tourist, in the field of contemporary artistic creation. Added to this notion is that of residence, very used in the artistic context - residency -, which is here to question. The tourist-resident-dweller artist implements poetic and political ways of being and acting and thus proposes a certain wisdom of dwelling.

\section{INDEX}

Keywords : Francis Alÿs, dwelling, living, tourist, walking, play, practice, poetics, interaction, ecosophy

Mots-clés : Francis Alÿs, habiter, résider, touriste, marcher, jeu, pratique, poétique, interaction, écosophie

\section{AUTEUR}

\section{PASCALE RIOU}

ATER - Université Lumière Lyon 2

Chercheuse associée - LARHRA - UMR 5190

Discipline : histoire de l'art

Adresse électronique : pascale.riou@hotmail.fr 